\title{
HORSESHOE KIDNEY WITH HYDRONEPHROSIS - TALES OF THE UNEXPECTED
}

\author{
M. Nikolova, R. Krasteva, B. Bogov, T. Tsocheva, VI. Milenova, K. Petkov, N. Chernev \\ University Hospital Alexandrovska, Medical University - Sofia, Bulgaria
}

\begin{abstract}
Horseshoe kidney is an inborn renal fusion anomaly. It is frequently associated with renal stones and infections. The diagnosis is made using imaging methods - ultrasound, $X$-ray of the abdomen and intravenous pyelography, computed tomography (CT), magnetic resonance imaging (MRI) and radionuclide investigations. The diagnosis sometimes is hard, especially when other abnormalities are present, i.e. hydronephrosis, nephrolithiasis, stenosis of the ureteropelvic junction, etc. The authors present a male patient with horseshoe kidney and unilateral hydronephrosis due to obstruction of the ureteropelvic junction and discuss the diagnosis of horseshoe kidney and the diagnostic approach in such patients.
\end{abstract}

Key words: horseshoe kidney, hydronephrosis, diagnosis, abdominal ultrasound, renal imaging

Correspoding author: Milena Nikolova, MD, PhD, Clinic of Nephrology, University Hospital Alexandrovska, Medical University, 1 G. Sofiyski St., 1431 Sofia, Bulgaria, e-mail: milena_i_dani@abv.bg

\section{INTRODUCTION}

Horseshoe kidney (HSK) is one of the most common inborn renal abnormalities, observed in 1:400-1:600 newborns $[1,2,3]$. It tends to have marked variability in the structure, position, blood supply and drainage of the fused kidneys. Usually, the lower poles of both kidneys are connected by a fibrous or parenchymatous isthmus (i.e., bridge) and rarely the upper poles are connected. The kidneys can be rotated and ureters - malpositioned (frequently with obstruction of the ureteropelvic junction) as can be the renal vessels. HSK can be accompanied by other renal and extrarenal abnormalities and can be complicated with renal stones, infections and renal tumors $[1,4,5,6]$. If not complicated by urinary tract infections or stones, this anomaly usually remains undiagnosed [2].

\section{CLINICAL CASE}

A 40-years-old male patient was admitted to the Clinic of Nephrology in June 2016 for diagnostic evalua- tion of right-sided hydronephrosis discovered during ultrasound examination of the abdomen performed for right-sided orchiepidydimitis. The patient reported mild discomfort in the right flank area. He had history of arterial hypertension with good control on bisoprolol $2.5 \mathrm{mg}$ a day. He also had past history of appendectomy and family history of arterial hypertension.

The physical examination at the admission revealed a palpable painless round formation in the right abdominal flank approximately $15-16 \mathrm{~cm}$ in diameter. Arterial blood pressure was $130 / 80 \mathrm{~mm} \mathrm{Hg}$. The rest of the physical examination was unremarkable.

The clinical laboratory investigations at the admission revealed normal ESR, blood count, liver enzymes, blood glucose, calcium and phosphate levels, serum creatinine $111 \mathrm{mcmol} / /$, estimated creatinine clearance of $99.6 \mathrm{ml} /$ min, urea $5.4 \mathrm{mmol} / \mathrm{l}$, urinalysis was unremarkable, urine sediment revealed several white blood cells per power field. The urine cultures showed no bacterial growth.

The abdominal ultrasound examination (figure 1) revealed hyperechogenic liver, normal image of 
the spleen, pancreas, urinary bladder and prostatic gland. The right kidney was enlarged $-170 \mathrm{~mm}$, with the image of giant hydronephrosis of long duration anechogenic dilated pelvis and renal calyces and renal parenchyma of 7-8 $\mathrm{mm}$ between the anechogenic zones. The left kidney was also enlarged $-150 \mathrm{~mm}$, with normal parenchyma and parenchymal "bridge" connecting it to the right kidney. The lower pole of the left kidney was elongated and tapered appearing to be curved away from the transducer (boomerang-like shape), suggesting the presence of HSK.

After the abdominal ultrasound examination an intravenous pyelography was performed (figure 2). The plain abdominal $\mathrm{X}$-ray revealed normal position of both kidneys. After the intravenous administration of contrast medium the right kidney remained "silent" no excretion of contrast medium. The left kidney excreted the contrast medium on minute 10 with double pelvis - the upper part appearing normal, and lower part located below at the level of L4-L5. Both pelvic structures to the left were drained by a single left ureter. The intravenous pyelogram was suggestive of a single S-shaped kidney to the left with absent or "silent" right kidney.

The abdominal CT revealed HSK with old hydronephrosis to the right due to stenotic obstruction of the right ureteropelvic junction and double left pelvis. Unfortunately, we lack the images of this investigation.

After the abdominal CT the patient was eventually diagnosed with combined urological anomaly: horseshoe kidney with impaired function of the right kidney due to stenosis of the ureteropelvic junction and a longstanding hydronephrosis with consumption of renal parenchyma, along with double left renal pelvis with single draining ureter. Subsequently the patient was referred to urologist for further treatment and evaluation for nephrectomy to the right.

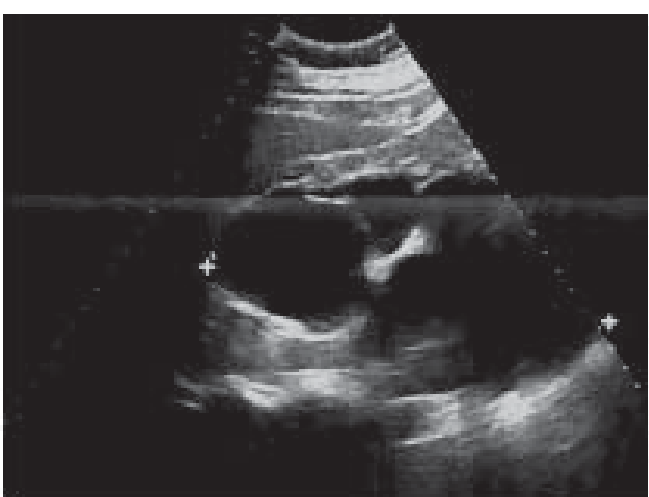

A

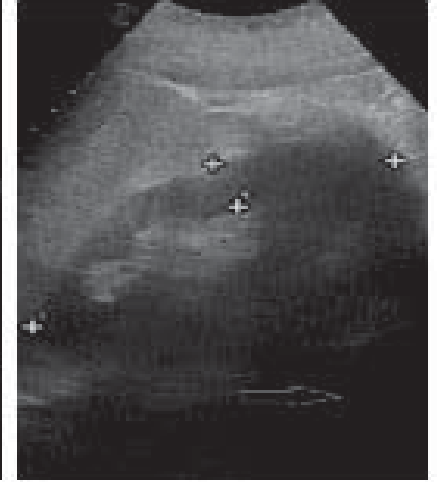

B

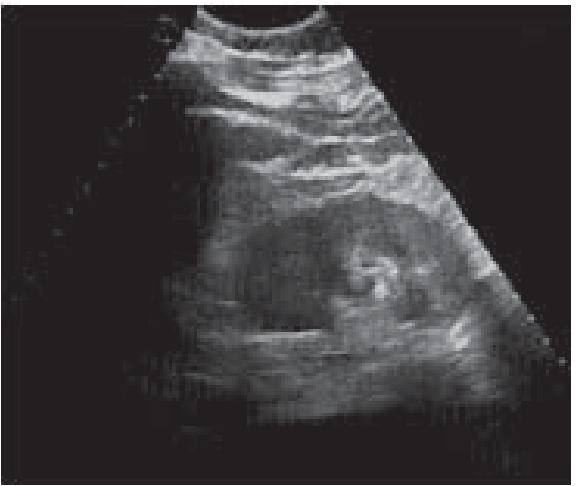

C

Fig. 1. Abdominal ultrasound examination. (A) Right kidney - hydronephrosis. (B) Left kidney - note the elongation and tapering of the lower pole making the kidney curved away from the transducer (arrow), suggesting the presence of HSK. (C) Parenchymal "bridge" connecting both kidneys (isthmus)

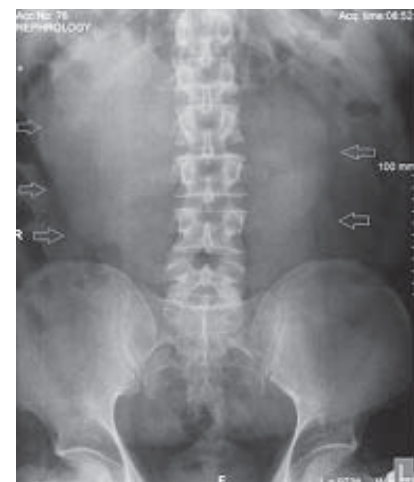

A

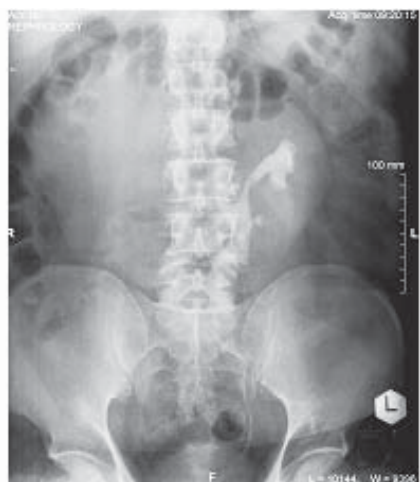

B

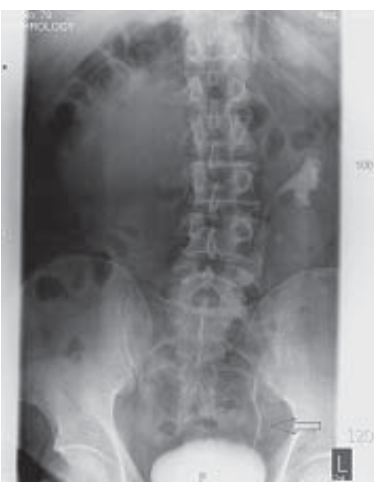

C

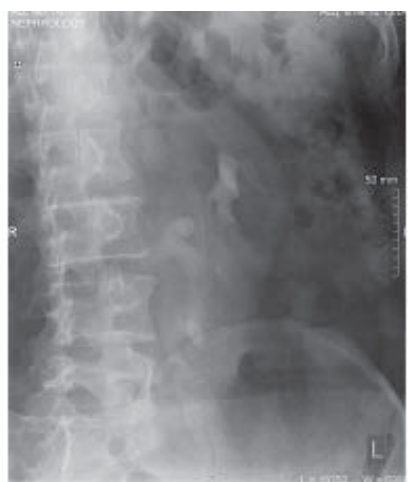

D

Fig. 2. Intravenous pyelogram. (A) Plain X-ray of the abdomen revealing normal position of both kidneys with well visible renal contours (arrows). (B) After the intravenous administration of contrast medium - the right kidney remained "silent" - no excretion of contrast medium. The left kidney excretes the contrast medium on minute 10 with double pelvis - the upper part appearing normal, and lower part is located below, at the level of L4-L5. (C) Both pelvic structures to the left were drained by a single left ureter (arrow). (D) The same image of a double left renal pelvis on targeted X-rays in standing position 


\section{DISCUSSION}

HSK is the most common congenital fusion abnormality of the urinary tract. It is observed in 1:400-600 live births, more frequently in men (male to female ratio approximately $2: 1)[1,2,3]$. HSK usually combines three distinct anomalies: renal ectopia, malrotation of the kidneys and renal vascular abnormalities [1]. In the majority of cases the kidneys are connected by an isthmus located at their lower poles (a Ushaped kidney), and less frequently the isthmus connects to the upper poles, forming an "inverted" HSK $[1,2]$. Horseshoe kidney represents a defect of renal ascending combined with fusion defect. It starts between the week 4 and 6 of gestation when the lower (inferior) part of the metanephric blastema fuses before the kidneys ascend and rotate. The fused tissue is the isthmus and it prevents the kidneys from ascending and rotating due to the resistance it exerts as it reaches the inferior mesenteric artery. HSK may be associated with other urinary tract and extrarenal abnormalities, including stenosis of the ureteropelvic junction, double pelvis, abnormalities of the urinary bladder, supernumerary kidneys with horseshoe elements, undescended testis and hypospadias, septate vagina; skeletal, cardiovascular, gastrointestinal and central nervous system malformations, chromosomal abnormalities (including Patau and Gardner syndromes, Down and Turner syndromes) [1-6]. Moreover, HSK has been found even more frequently in autopsy examinations of children and infants, suggesting the high risk of mortality in young age, probably due to severe associated organ and/or chromosomal abnormalities [1].

HSK can be complicated by the formation of kidney stones, hydronephrosis (due to stenosis of the ureteropelvic junction, malrotation and/or malposition of the kidneys and ureters, obstruction by stones), urinary tract infections and tumors (Wilms' tumor usually in the isthmus, transitional cell carcinoma, oncocytoma, carcinoid tumors) [1-6].

In the adult population HSK is usually asymptomatic and can be discovered accidentally during imaging studies for other reason or for renal stones or urinary tract infections [3]. The ultrasound image is very typical - low-lying and malrotation of one or both kidneys, anterior position of renal pelvices with or without hydronephrosis/pyelectasia, boomerang-shaped kidneys with elongation, poorly-defined lower pole curving away from the transducer [3]. The isthmus may not be clearly seen on ultrasound investigation [3]. The intravenous pyelogram and the contrast CT/ MRI may reveal the typical U-shaped kidney with two ureters, and the CT/MRI reveal changes in renal vessels [1]. In cases of hydronephrosis CT/MRI and radionuclide assays are very valuable, as they reveal both renal structure and excretion.

In our patient we observed unilateral hydronephrosis with palpable formation in the right flank, boomerangshaped left kidney suggesting HSK, and X-ray data for a silent right kidney, suggesting complete obstruction of the urinary flow to the right. The presence of a single left ureter to the left does not confirm the suspected S-shaped left kidney (homolateral fusion abnormality) but does suggest double pyelo-calical system to the left with a sigle draining ureter. The CT confirmed the presence of HSK with unilateral hydronephrosis (right-sided) due to obstruction of ureteropelvic junction and double left renal pelvis. Therefore, the patient had three urinary tract abnormalities: HSK, stenosis of the right ureteropelvic junction and double left pelvis. The combined efforts of several imaging methods led to the correct diagnosis. The ultrasound could not detect the cause of the rightsided hydronephrosis but suggested the presence of HSK, the intravenous pyelogram did not reveal the right kidney and suggested S-shaped left kidney, and the contrast CT revealed all three abnormalities and explained the palpable formation in the right flank. Therefore, we would like to underline the importance of the thorough physical examination and of the combined use of multiple imaging methods for the detection of urinary tract abnormalities.

\section{REFERENCES}

1. Natsis K, Piagkou M, Skotsimara A, et al. Horseshoe kidney: a review of anatomy and pathology. Surg Radiol Anat. 2014 Aug;36(6):517-26.

2. Rao K EV, Battula SR. Horseshoe kidney: a review article. Int J Res Med Sci. 2015;3(11):3004-3007.

3. Strauss S, Dushnitsky T, Peer A, et al. Sonographic features of horseshoe kidney: review of 34 patients. J Ultrasound Med. 2000;19:27-31.

4. Kaakaji Y, Pfister RC. Horseshoe kidney with severe congenital hydronephrosis (ureteropelvic junction obstruction). AJR. 1998;171:829.

5. Krishnan B, Truong LD, Salek G, et al. Horseshoe kidney is associated with an increased relative risk of primary renal carcinoid tumor. J Urol. 1997;157:205-2066.

6. Rodríguez-Covarrubias F, Gómez X, Valerio JC, Lome-Maldonado C, Gabilondo F. Carcinoid tumor arising in a horseshoe kidney. Int Urol Nephrol. 2007;39(2):373-376. 